\section{Vol. 70, No. 21}

In the report "Racial and Ethnic Disparities in Breastfeeding Initiation - United States, 2019," on page 774, the last sentence should have read, "Implementation of evidencebased maternity care policies and practices supportive of breastfeeding and targeted breastfeeding programs focusing on populations at highest risk for low breastfeeding initiation might help reduce racial/ethnic disparities in breastfeeding initiation, improve infant nutrition, and reduce maternal and infant morbidity."

\section{Vol. 70, No. 32}

In the report "West Nile Virus and Other Domestic Nationally Notifiable Arboviral Diseases - United States, 2019," on pages 1072 and 1073, in Table 2, rates should have been $<\mathbf{0 . 0 1}$ for the following viruses and U.S. Census divisions: La Crosse virus for the West North Central Division; Eastern equine encephalitis for the South Atlantic division; and St. Louis encephalitis for the United States (total) and the West South Central division. 